\title{
Determination of the Luminosity at the LHC Experiments
}

\section{Massimiliano Ferro-Luzzi*}

CERN, Geneva, Switzerland

E-mail: Massimiliano.Ferro-Luzzi@cern.ch

A report is given on the first series of experiments that were performed at the LHC in 2009 and 2010 to obtain first luminosity calibration measurements for each LHC Interaction Point at the zero-momentum frame energies of $\sqrt{s}=0.9$ and $7 \mathrm{TeV}$. Two methods were used, the van der Meer scan method and the beam-gas imaging method, which are briefly explained.

35th International Conference of High Energy Physics - ICHEP2010, July 22-28, 2010

Paris France

*Speaker. 


\section{Introduction}

The ability to measure luminosity on an absolute scale is of general interest in colliding-beam particle physics experiments at storage rings. It allows to determine the absolute cross-section value of reaction processes and to quantify the performance of the machine. For instance, at the CERN Large Hadron Collider (LHC), which has started physics operation in the year 2009, the LHC experiments can use precise cross-section measurements to constrain QCD-based models of $p p$ interactions and to detect or quantify new phenomena due to physics beyond the Standard Model of particle physics. In some cases, the required accuracy on the absolute value of the cross section lies in the range $1-5 \%$.

The luminosity $L$ for two counter-rotating bunches (here, 1 stands for Beam1 and 2 for Beam2) with time- and position-dependent density functions $\rho_{1}(\mathbf{x}, t)$ and $\rho_{2}(\mathbf{x}, t)$ is given by

$$
L=f N_{1} N_{2} 2 c \cos ^{2}(\phi / 2) \int \rho_{1}\left(x-\delta_{x}, y-\delta_{y}, z, t\right) \rho_{2}(x, y, z, t) d^{3} x d t
$$

when Beam1 is transversely displaced by $\delta=\left(\delta_{x}, \delta_{y}, 0\right)$ relative to Beam2. Here we have assumed ultra-relativistic particles, a crossing angle $\phi$ and we defined $f$ the revolution frequency, and $N_{i}$ the total number of protons in the bunch of beam $i$ that contribute to the luminosity. The bunch particle densities $\rho_{1}(\mathbf{x}, t)$ and $\rho_{2}(\mathbf{x}, t)$ are normalized such that their individual integrals over full space are unity. In the early phase of the LHC 2009 and 2010 runs, a series of experiments were carried out to perform a first luminosity calibration measurement at each Interaction Point (IP). Two methods were employed: the "van der Meer scan" method (vdM) and the "beam-gas imaging" method (BGI).

In the vdM method [1] a reaction rate is measured as a function of the displacements of the beams along the two transverse axes. For any given process of cross section $\sigma_{\mathrm{P}}$, the reaction rate is $R\left(\delta_{x}, \delta_{y}\right)=\sigma_{\mathrm{P}} L\left(\delta_{x}, \delta_{y}\right)$. When integrated over the displacements, the measured rate gives the cross section (independent of the beam overlap integral). If the density distributions can be factorized, then two scans are sufficient to obtain the cross section: one along a constant $y$-displacement $\delta_{y 0}$ and one along a constant $x$-displacement $\delta_{x 0}$. It can be shown that, even in the presence of a non-zero full crossing angle $\phi[2]$,

$$
\sigma_{\mathrm{P}}=\frac{\int R\left(\delta_{x}, \delta_{y 0}\right) d \delta_{x} \cdot \int R\left(\delta_{x 0}, \delta_{y}\right) d \delta_{y}}{f N_{1} N_{2} \cos (\phi / 2) R\left(\delta_{x 0}, \delta_{y 0}\right)} .
$$

It is assumed that effects due to bunch evolution during the scans (shape distortions or transverse kicks due to beam-beam effects, emittance growth, bunch current decay), effects due to the transverse bunch distribution tails and effects of the absolute length scale calibration against magnet current trims either are negligible or can be corrected for.

In the BGI method, the beams are left untouched and interactions between beam protons and nuclei of the residual gas are detected. Reconstructing such distinctive vertices allows one to obtain an image of the transverse bunch profile along the beam trajectory. From the two individual bunch profiles, it is then possible to reconstruct the beam-overlap integral. The simultaneous reconstruction of the luminous region with the vertex detector can also be used to further constrain the beam parameters. 
For this method a vertex resolution is needed that is comparable or smaller than the transverse beam sizes. Furthermore, the acceptance of the vertex detector must be suitable for detecting beamgas interactions in the vicinity of the IP. For LHCb, at a residual gas pressure of approximately 2 . $10^{-9}$ mbar (dominated by hydrogen gas, $\mathrm{H}_{2}$ ), a beam-gas interaction rate of about $R \approx n d \sigma_{p p} f N \approx$ $0.02 \mathrm{~Hz}$ is obtained per bunch of $N=10^{10}$ protons, for free (i.e. without any attempt to increase the rate). Here, we have used $n \approx 10^{8} \mathrm{~cm}^{-3}$ for the density of gas protons, $\sigma_{p p} \approx 37 \mathrm{mb}$ for the $p p$ inelastic cross section of $7 \mathrm{TeV}$ protons on protons at rest $(\sqrt{s}=81 \mathrm{GeV})$ and $d \approx 50 \mathrm{~cm}$ for the length over which beam-gas interaction can be reconstructed by the LHCb vertex detector (VELO). At the LHC injection energy $(450 \mathrm{GeV})$, the cross section is slightly reduced, $\sigma_{p p} \approx 33 \mathrm{mb}$.

Compared to the vdM method, the disadvantage of a small rate (which, however, could be increased by controlling the residual vacuum pressure) is balanced by the advantages that (i) the method is non-disruptive, the beams do not move, and (ii) the method can be applied at any time, while taking physics data.

\section{The measurements}

Pioneering measurements were carried out at LHCb, in December 2009, at a $p p$ center-ofmass energy of $\sqrt{s}=0.9 \mathrm{TeV}$ using the BGI method. In April-May 2010, more measurements were carried out at the beam energy of $3.5 \mathrm{TeV}(\sqrt{s}=7 \mathrm{TeV})$ with both the BGI method at IP8 [2] and the vdM method at all IP [3]. The beams were squeezed to $\beta^{*}=2 \mathrm{~m}$, corresponding to an individual beam size of about $45 \mu \mathrm{m}$ (assuming nominal normalised transverse emittance $\varepsilon=3.75 \mu \mathrm{m}$ ). No external crossing angle was applied, which resulted in a zero net crossing angle at IP1 and IP5. However, due to the presence of a spectrometer dipole, a net crossing angle of 280 (540) $\mu \mathrm{rad}$ in the vertical (horizontal) plane was present at IP2 (IP8). The vdM scans were done in IP5/CMS (LHC fills 1058 and 1089) IP1/ATLAS (1059 and 1089), IP8/LHCb (1059) and IP2/ALICE (1090), with either two $(1058)$ or one $(1059,1089,1090)$ colliding bunch pair. The bunch population was either $\sim 1 \cdot 10^{10}(1058,1059)$ or $\sim 2 \cdot 10^{10}(1089,1090)$.

For illustration, fig. 1 (left) shows an online display of the beam position monitor signals at IP8 while performing vdM scans for LHCb in April 2010. A first scan was made horizontally by moving the two beams by the same mount in opposite directions (red and green symbols). A second scan was done similarly in the vertical direction (blue and purple symbols). A second series of scan scans was then made with only Beam1 moved across Beam 2 along the two axes. The raw level-0 trigger calorimeter rate measured by the $\mathrm{LHCb}$ detector while performing the $\mathrm{vdM}$ scans is shown in fig. 1 (right). The red points indicate the rate when the beam positions were back to their original position, as before starting the scans. The dashed line is a fit to these points indicating the luminosity decrease across the duration of the vdM scans.

The BGI method was applied by LHCb over several LHC fills. An example image of the two LHC beams is shown in fig. 2 which was obtained by plotting the positions of the reconstructed beam-gas interaction vertices at IP8. In this case, the beam energy was $450 \mathrm{GeV}$. The lines are the results of a linear fit to the vertex positions. From the data a measurement of the beam crossing angle can also be extracted.

The vdM and BGI methods provide a measurement of the beam-overlap integral. For determining the luminosity, it is crucial to obtain separately a measurement of the bunch population 

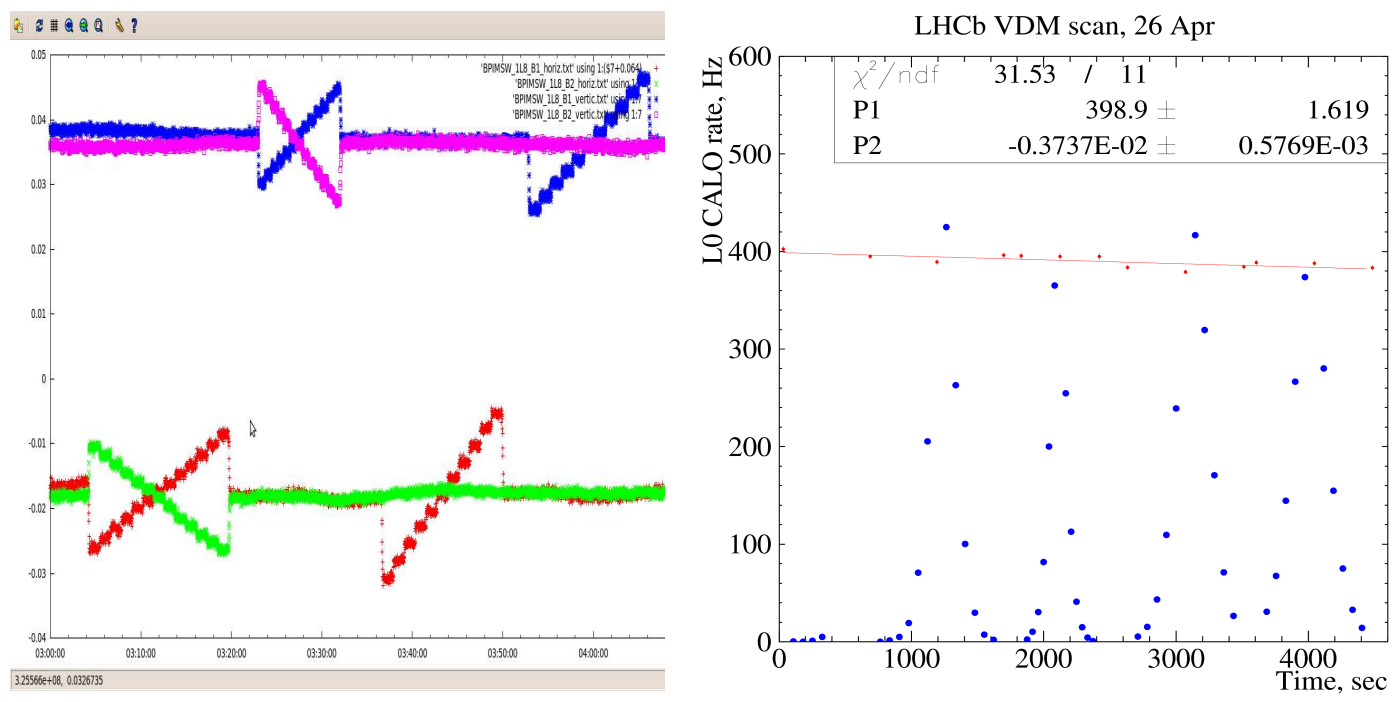

Figure 1: Left: Online display of the beam position monitor signals at IP8 while performing vdM scans in April 2010. Red (green) symbols are for the horizontal position of Beam1 (Beam2), while blue (purple) symbols are for the vertical position of Beam1 (Beam2). Right: Raw level-0 calorimeter rate measured by the LHCb detector while performing the vdM scans in April 2010. See text.

product $N_{1} N_{2}$ appearing in eq. 1.1. The bunch intensity measurement for the LHC ring was provided by eight current transformers [4], two DC current transformers (DCCT) and two fast beam current transformers (FBCT) installed on the vacuum chamber of each circulating beam. The DCCT gave a measurement of the total current circulating in each ring, irrespective of the time structure of the beams. The FBCT gave a measurement of the bunch populations in each of the 3564 nominal (25 ns) slots of each beam.
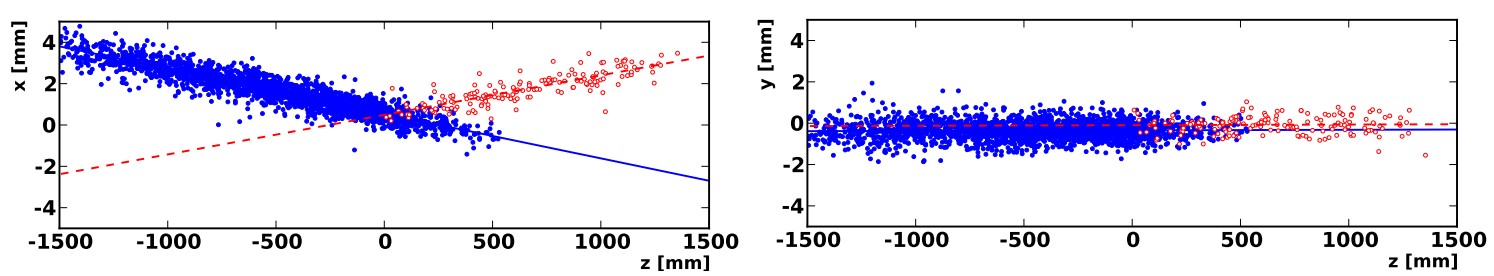

Figure 2: Image of the colliding LHC beams at IP8 obtained by vertex reconstruction of beam-gas interactions with the LHCb VELO. Blue dots: Beam1. Red circles: Beam2. The lines are the results of a linear fit to the vertex positions. The full crossing angle of $\phi \approx 4.2 \mathrm{mrad}$ due to the LHC dipole spectrometer is visible in the $x-z$ plane.

\section{Preliminary analysis results}

Each LHC experiment carried out an analysis of the vdM scans to extract an absolute luminosity. In the measurements presented here, the bunch shape evolution was obtained from wire scanner data taken at different moments, before, during and after the experiments. The vdM scan data were 
corrected for a small emittance growth (typically $<2 \%$ ). The current decay during the scans was measured with the LHC beam current transformers and corrected for (also typically $<2 \%$ ). The scans extended up to $\sim 0.27 \mathrm{~mm}$ separation between the two beams (i.e. $\sim 6$ nominal beam $\sigma$ ). The length scale was calibrated using imaging of the luminous region by vertex reconstruction for different positions of the the luminous region. In a first preliminary analysis of the LHC beam current transformers data, shortly after the luminosity calibration experiments, a conservative estimate of the current normalisation was made, which became the dominant uncertainty $(\sim 10 \%$ as opposed to 3 to $5 \%$ for the total contribution of other sources of systematic uncertainties).

The individual bunch populations $N_{i}$ (of each beam) were obtained assuming that the sum of populations of all nominally filled bunches $i=1, \ldots n$, as measured by the FBCT, was equal to the value of the total beam population obtained from the DCCT $N_{\text {tot }}$. The amount of possible "ghost" charge, defined as the charge not visible to the FBCT, was estimated from detector data by the experiments (in particular from open beam-gas triggers in $\mathrm{LHCb}$ ) and resulted to be relatively small ( $\sim 1 \%$ or less of the total charge) compared to the total uncertainty on the bunch currents. In the preliminary analysis the ghost charge was neglected. The total intensity was taken from $N_{\text {tot }}=\alpha \cdot S_{\text {DCCT }}$, where $\alpha$ is the absolute scale factor of the DCCT (elementary charges/V) and $S_{\text {DCCT }}$ the signal measured by the DCCT (V) after correcting for a baseline offset.

The uncertainty on the absolute bunch intensity was dominated by that on the overall accuracy of the DCCT. The DCCT readings may be distorted by various effects [4] such as temperature drifts, electromagnetic pick-up in cables, mechanical vibrations of the transformer assembly, etc. For the beam intensities and with the sparse bunch pattern used in the April-May $2010 \mathrm{vdM}$ scans, the dominant uncertainty is associated with the absolute scale accuracy of the DCCT and its baseline drifts.

The time history of the DCCT baselines revealed fluctuations on a wide range of time scales. An early estimate of these fluctuations suggested that with the automated averaging algorithm applied to the DCCT data at that time, the DCCT baselines measured before and after each fill were each affected by an equivalent peak-to-peak (or "envelope") uncertainty of $\pm 1 \cdot 10^{9}$ protons in total beam intensity. This was conservatively translated into a peak-to-peak uncertainty of $\pm 2 \cdot 10^{9}$ protons on the total beam current reported by the DCCT in the period between the two baseline measurements bracketing the fill under study. This led to the following estimate of the uncertainty on the measured bunch intensity $N_{i}$ associated with the DCCT baseline subtraction: $\Delta N_{i} / N_{i}=$ $\pm 2 \cdot 10^{9} / N_{\text {tot }}$, where $\Delta N_{i}$ is the peak-to-peak uncertainty on $N_{i}, n$ is the total number of bunches per beam, and $\left\langle N_{i}\right\rangle$ is the mean charge per bunch. It should be stressed that $\Delta N_{i}$ is a peak-to-peak or "tolerance" uncertainty, in the sense that it is meant to cover the full possible range of measurement errors; in other words, the true value of $N_{i}$ may lie anywhere in the interval $N_{i} \pm \Delta N_{i}$, but not outside. Interpreting $\Delta N_{i}$ as a one-sigma error would therefore be an overestimate. Under the assumption of a uniform error distribution of half width $\Delta N_{i} / N_{i}$, the fractional one-sigma uncertainty associated with DCCT baseline drifts can be estimated by $\sigma_{N_{i}} / N_{i}=\Delta N_{i} /\left(\sqrt{3} N_{i}\right)=1.15 \cdot 10^{9} / N_{\text {tot }}$. This led, for example, to a bunch-charge uncertainty (for each beam) of $\sigma_{N_{i}} / N_{i}=5.8 \%$ in the case of a beam with two circulating bunches containing $1 \cdot 10^{10}$ protons each.

In the preliminary analysis, the DCCT current offset variations of the two beams were conservatively estimated and treated as fully correlated errors, while the FBCT relative population uncertainties were neglected. The total uncertainties on the bunch population products were calculated 
(again, conservatively) from the linear sum of the DCCT baseline and scale factor contributions. This resulted in total bunch product population uncertainties ranging from 8 to $14 \%$, depending on the fill.

In view of the magnitude of the DCCT baseline uncertainties, a more precise quantitative characterization of these errors and of their degree of correlation between the two beams is currently being finalized. First results indicate that the uncertainties may be reduced by about a factor two [5]. Furthermore, a comparison of the the FBCT relative bunch populations to the ATLAS BPTX signals (which also provide a measurement of the relative bunch populations) is now under way to better evaluate the accuracy of the relative bunch population results. The amount of ghost charge is also being evaluated more precisely and will be taken into account.

\section{Summary}

In this work we reported on the first luminosity normalisation measurements performed at the LHC in December 2009 and April-May 2010. These calibration experiment were based on two methods: the van der Meer scan method and the beam-gas imaging method. The main uncertainty stemmed from the determination of the bunch population product as obtained from a preliminary analysis of the LHC beam current transformers data. This analysis resulted in total uncertainties for the bunch population products ranging from 8 to $14 \%$, depending on the LHC fill. A more detailed analysis is currently being finalized which should produce bunch population product uncertainties about a factor two smaller.

\section{Acknowledgements}

We would like to thank all LHC colleagues who made these measurements possible, as well as our colleagues from the LHC Collaborations. Special thanks to G. Anders, P. Baudrenghien, N. Bacchetta, V. Balagura, C. Barschel, D. Belohrad, H. Burkhardt, S. Cooper, G. Franzoni, C. Gabaldon, M. Gagliardi, J.J. Gras, V. Halyo, B. Heinemann, P. Hopchev, A. Hunt, W. Kozanecki, S. Kwan, M. Ludwig, D. Marlow, P. Odier, K. Oyama, S. Pagan Griso, J. Panman, T. Pauly, D. Stickland, S. Thoulet, S. White, J.C. Yun and M. Zanetti.

\section{References}

[1] "Calibration of the effective beam height in the ISR", S. van der Meer, ISR-PO/68-31, 1968 (CERN), and "Absolute Luminosity from Machine Parameters", H. Burkhardt, P. Grafström, LHC-PROJECT-Report-1019; CERN-LHC-PROJECT-Report-1019.

[2] V. Balagura, private communication, and "Luminosity measurements in the first LHCb data", V. Balagura, in proc. of the 2010 Rencontres de Moriond (QCD), La Thuile, 2010.

[3] See for example in "First Luminosity Scans in the LHC”, S.M. White et al., Proc. IPAC'10.

[4] “The 2010 LHC DC BCT measurement system [...]”, P. Odier, J.-J. Gras, M. Ludwig, S. Thoulet, LHC-Project-Note-432, and “The 2010 LHC ring Fast BCT measurement system [...]”, D. Belohrad, J.-J. Gras, M. Ludwig, LHC-Project-Note-433.

[5] "LHC bunch current normalisation for the April-May 2010 luminosity calibration experiments", G. Anders et al., CERN-PH note in preparation. 